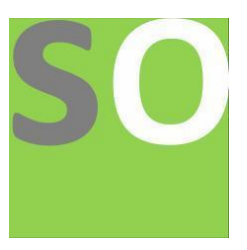

Article title: Quantum certainty mechanics

Authors: Muhammad Yasin[1]

Affiliations: Researcher in Bangladesh[1]

Orcid ids: 0000-0001-7303-8390[1]

Contact e-mail: md.yasin78654@gmail.com

License information: This work has been published open access under Creative Commons Attribution License http://creativecommons.org/licenses/by/4.0/, which permits unrestricted use, distribution, and reproduction in any medium, provided the original work is properly cited. Conditions, terms of use and publishing policy can be found at https://www.scienceopen.com/.

Preprint statement: This article is a preprint and has not been peer-reviewed, under consideration and submitted to ScienceOpen Preprints for open peer review.

Links to data: Muhammad Yasin

DOI: 10.14293/S2199-1006.1.SOR-.PPW38QY.v1

Preprint first posted online: 10 January 2021

Keywords: Quantum mechanics, Uncertainty principle, Bhor's atomic model, Quantum certainty mechanics, Simultaneous measurement, Certain measurement, Plank's radiation law, Relativity theory 


\title{
Quantum Certainty Mechanics
}

\author{
Muhammad Yasin \\ Researcher in Bangladesh \\ e-mail:md.yasin@ymcontents.com
}

\begin{abstract}
1.Abstract:Quantum certainty mechanics is a theory for measuring the position and momentum of a particle. Mathematically proven certainty principle from uncertainty principle, which is basically one of the most important formulas of quantum certainty mechanics theory. The principle of uncertainty can be proved by the principle of certainty and why uncertainty comes can also be proved. The principle of certainty can be proved from the theory of relativity And in the uncertainty principle equation, the principle of certainty can be proved by fulfilling the conditions of the principle of certainty by multiplying the uncertain constant with the certain values of momentum-position and energy-time. The principle of certainty proves that the calculation of $\theta \geq \pi / 2$ between the particle and the wave involved in the particle leads to uncertainty. But calculating with $\theta=0$ does not bring uncertainty. Quantum certainty mechanics has been established by combining Newtonian mechanics, relativity theory and quantum mechanics. Quantum entanglement can be explained by protecting the principle of conservation of energy.
\end{abstract}

2.Keywords: quantum mechanics, uncertainty principle, quantum entanglement, planck's radiation law, bohr's atomic model, certainty mechanics, quantum measurement.

\section{Introduction}

In 1927 Heisenberg has invented the uncertainty principle.The principle of uncertainty is, "It is impossible to determine the position and momentum of a particle at the same time."The more accurately the momentum is measured, the more uncertain the position will be.Just knowing the position would make the momentum uncertain. Einstein was adamant against this principle until his death.He thought that particles have some secret rules. Einstein thought, "The uncertainty principle is incomplete. There is a mistake somewhere that has resulted in uncertainty. Many did not accept Einstein then. But I'm sure Einstein was right then, there are secret rules for particles. Heisenberg's uncertainty principle is also $100 \%$ correct.There are waves with the particle (The Broglie's wave).If we calculate $\theta \geq \pi / 2$ between waves and particles then uncertainty will appear. But if we calculate $\theta=0$ then there is no more uncertainty. The purpose of this research paper is to establish Einstein's concept of the secret law, that is, to establish the theory of certainty, to establish the relationship between the principle of certainty and the principle of uncertainty, and to explain why uncertainty occurs. This article will have a total of four formulas including the certainty policy. The formulas together are known as certainty mechanics. Bohr's angular momentum can be proved by certainty mechanics, Bohr's energy radiation formula can be proved, Max Planck's quantum theory and Heisenberg's law of uncertainty can be proved by certainty mechanics. The formulas are established by protecting the conservation law of energy. The complex problems of quantum mechanics can be solved by certainty mechanics. The 
essence of the principle of certainty is that at the same time it is possible to accurately determine the position and momentum of electrons. The more accurately the position is known, the more accurately it is possible to determine the momentum.Photons are thrown at electrons while observing electrons by laboratory equipment. As a result, the position and momentum of the electron change. The principle of certainty is established by calculating the energy of that photon.There is a relationship between the principle of certainty and the principle of uncertainty. The principle of uncertainty can be proved by the principle of certainty just as the principle of certainty can be proved by the principle of uncertainty.

4. Formulas proved by certainty mechanics: The basic formulas of quantum mechanics can be proved by certainty mechanics.Let's take a look at all the formulas that can be proved from this theory.

- Bohr's formula for angular momentum.

- Planck's quantum formula.

- Bohr's proposal regarding energy radiation.

- "Uncertainty Principle".

5. Evidence of the law of certainty mechanics: There are 4 formulas in certainty mechanics which are based on quantum mechanics. Certainty mechanics is a combination of Newtonian mechanics, quantum mechanics and the theory of relativity. The "principle of certainty" comes from truthful relativity theory .

(i) Evidence of the second law: from the particle wave duality of The Broglie,

$$
\begin{aligned}
& \lambda=\frac{h}{p} \\
& =>p=\frac{h}{\lambda} \\
& =>p=\frac{h}{c} f \quad\left(\lambda=\frac{c}{f}\right) \\
& =>p=\frac{h}{c} f
\end{aligned}
$$

In this formula there is a relationship between momentum and frequency. This is as true for electrons as it is for large objects. The wavelength $\lambda$ is involved in its matter wave whenever the object starts moving with $\mathrm{P}$ momentum. When an object gains momentum, waves are created due to the momentum, or we can say that Broglie's waves are involved.We are not waves, waves are created when we move. Thus there is a relation between the frequency and the wave created due to the momentum of the object.If a boat runs on the river, waves will be created. The shape of the wave depends on the momentum of the boat. Similarly how waves and frequencies will be created when an object moves with momentum will depend on the momentum of the object. From Equation 1 it is seen that the momentum of an 
object and the frequency of the waves created or involved due to the momentum are proportional .

If the momentum of an object changes, the frequency of the waves involved due to the momentum will also change .

$$
\therefore p_{1}=\frac{h}{c} f_{1}
$$

Now, if the momentum changes from $p_{1}$ to $p_{2}$, the momentum will be,

$$
\therefore p_{2}=\frac{h}{c} f_{2}
$$

So, the change of momentum ,

$$
\begin{aligned}
& d p=p_{2}-p_{1} \\
& =\frac{h}{c}\left(f_{2}-f_{1}\right) \\
& \therefore d p=\frac{h}{c} d f
\end{aligned}
$$

Now if the velocity of the particle is $v$, the form of the equation will be,

$d p=\frac{h}{v} d f$

That is, if the momentum of an object changes, the frequency of the wave created or involved due to the momentum will change .

Newton's second law is,

$$
F=\frac{d p}{d t}
$$

Now, let us set the value of $d p$ from equation 2 ,

$$
\begin{aligned}
& \therefore F=\frac{h}{c} \frac{d f}{d t} \\
& \because F=\frac{h}{c}\left(\frac{f_{1}-f_{2}}{d t}\right)
\end{aligned}
$$

If the velocity of the particle is $\mathrm{v}$, the formula will be,

$$
F=\frac{h}{v} \frac{d f}{d t}
$$

Here the initial velocity and end velocity of the object and the dt time difference is very fewer So we can write $v$ as constant. But the difference of the frequency of the waves involved at $\mathrm{dt}$ time is comparatively greater than df. However, the frequency difference (df) of the waves involved at $\mathrm{dt}$ time is relatively high. Suppose $A$ car with $P$ velocity is moving in a straight line. You are changing the momentum of the vehicle by applying $F$ force or you can say that by applying $\mathrm{F}$ force on the car you are changing the frequency of the waves involved due to its momentum. In this way it is possible to reconcile quantum mechanics with classical 
physics. If we calculate according to quantum mechanics then it can be seen that The energy of an electron changes as a result of the application of force $F$ by a photon. Which means that the application of $F$ force on an electron changes the frequency of the waves involved due to the momentum of the electron .The linguistic form of the second formula will be: -

"The rate of change of the frequency of the waves created or involved due to the $p$ momentum of an object or particle is proportional to the force applied to the object."

$F \propto \frac{d f}{d t}$

(ii) Proof of the first formula: Let us look at Equation No. 1 again.

$$
p=\frac{h}{c} f
$$

This equation can be written

$$
f=\frac{c}{h} p
$$

Thus it is seen from the equation that there is an unbroken relationship between the frequency of the wave created and the momentum. If the momentum is $p=0 \mathrm{kgms}^{-1}$ then the frequency of the wave involved will be $f=0 \mathrm{~Hz}$.Again if the momentum is a constant number then the frequency will be a constant number.If you do not apply force, the momentum will not change.Similarly, if the force is not applied, the frequency of the wave created due to the momentum of the object will not change.So if the force is not applied, the frequency of the wave created due to the momentum of the object will remain constant for life.So the first formula stands: -

"If no force is applied, the frequency of the waves involved ( created waves ) due to the momentum of the object will remain constant."

So if $F=0 N$ the frequency $f$ of the wave created due to the momentum of the object will be constant .

Again we get from the second formula,

Now let us calculate the external force $\mathrm{F}=0 \mathrm{~N}$.

$$
\begin{aligned}
& F=\frac{h}{c}\left(f_{1}-f_{2}\right) \\
& =>0=\frac{h}{c}\left(f_{1}-f_{2}\right)[F=0 N] \\
& \therefore f_{1}=f_{2}
\end{aligned}
$$

That is, the above equation also proves that if the force $F$ is not applied, the f frequency of the wave created (or involved) due to the momentum of the object will remain constant for life.

(iii) Evidence of third law: This law is similar to Newton's third law. Newton has applied his formula for point particles. But this formula applies to all object particles, including electrons. 
According to the conservation law of energy, energy is never destroyed. Is simply transformed from one form to another. The energy of an electron will change if a photon with $E=h v$ energy pushes $F$ force to the electron. As a result of the conservation law of energy the electron will give a reaction force to the photon. These action and reaction forces are equal but opposite.

$F_{1}=-F_{2}$

\section{(iv) Evidence of the fourth formula: The principle of certainty Method (1)-}

Heisenberg's uncertainty principle shows that the position and momentum of a particle cannot be measured accurately at the same time. The more accurately we measure one, the more uncertain the other will become. Einstein thought that uncertainty was not entirely right. There is a mistake that has led to uncertainty. The particles follow some secret rule.I will now extract that secret rule from this principle of certainty. One thing we have noticed in Heisenberg's formula is that light has to be emitted on electrons to determine their position. In order to determine the position accurately, it is necessary to emit high frequency light that means more light.But we are not bringing this matter in calculation, we are saying that light of $\lambda$ wavelength has to be shed. Light is a 'photon' particle. If the photon falls on the electron, it will push the electron with " $F$ " force. That is, light of $f$ frequency will push the electron.But we are not doing that calculation, we can accurately determine the momentum and position by calculating the external impact in the laboratory. If we bring $f$ frequency light in the calculation then the "certainty principle" will come.

The momentum of the particle and the wavelength of the involved wave from The Broglie's equation;

$$
\begin{aligned}
\lambda & =\frac{h}{m v} \\
\therefore p & =\frac{h}{c} f
\end{aligned}
$$

From this equation it is clear that when an object moves at a p momentum, waves are involved due to its momentum. Now imagine, an electron is moving at "p" momentum. The momentum of electrons will create a wave that will have a frequency $f$.

Suppose, if the electron moves with velocity $v$, crosses $x$ distance at $t$ time then the equation will be

$x=v t$

Now when the electron moves at a p momentum, the frequency of the wave (involved wave) will be created due to the momentum of the electron according to Equation 3. From the duality of The Broglie we know that waves are intertwined with electrons. Electrons have a velocity. The wave involved with the electron also has a velocity. The velocity of an electron is called the particle velocity and the wave velocity of the wave associated with the electron is called the phase velocity. But the problem is that the velocity of the wave attached to the electron is not equal to the velocity of the electron.The phase velocity is $V a=f \lambda$. And the velocity of the particle is $\mathbf{V}$.

$$
\therefore V=\frac{h}{m \lambda}
$$

The velocity of a particle and the velocity of a wave associated with a particle are not equal, but the velocity of a particle is equal to the velocity of a bunch of waves associated with a particle. Cluster velocity is - many waves travel in clusters when they are transmitted in the 
same direction. The velocity of this cluster wave is called cluster velocity. suppose cluster velocity is $\mathrm{Vg}$.

$$
\therefore V_{g}=\frac{d \omega}{d k}
$$

The relationship between cluster velocity and phase velocity

$$
V_{g}=v_{a}-\lambda \frac{d v_{a}}{d \lambda}
$$

Now the velocity of the particle and the velocity of the cluster are equal So, If $\mathbf{V g}=\mathbf{V}$

$$
\therefore V=v_{a}-\lambda \frac{d v_{a}}{d \lambda}
$$

Cluster velocity and particle velocity are equal but particle velocity and wave velocity are not equal. So Va will not be equal to V. But in this case a work can be done. Let's imagine the currencies of America and Bangladesh.According to the current market, 1 US dollar is equal to 85 Bangladeshi rupees. The value of 1 dollar is not 1 taka but one can represent another currency. What can be done with 10 dollars can be done with 850 rupees.

How far you can go with 1 dollar by car you can go the same distance in the same country with 85 taka.

$\mathrm{t}=$ taka

$\mathrm{T}=$ dollar

$\mathrm{V}=$ car's velocity

Now if I go with taka by car then I can write

$\mathrm{S}=\mathrm{Vt}$

Or if I go with dollar by car then I can write

$\mathrm{S}=\mathrm{VT}[\mathrm{T} \neq \mathrm{t}$ but we can represent $\mathrm{t}$ by $\mathrm{T}]$

(Compared here with two currencies of the same country)

It turns out that money or dollars can go the same distance.So we can represent $t$ instead of T.In exactly the same way we can represent the velocity of a particle with the velocity of a wave.

So here we put the value of $\mathrm{Va}$ instead of velocity $\mathrm{v}$ at $\mathrm{S}=\mathrm{vt}$.

$$
v=f \lambda
$$

From 4 no. And 5 no. equation-

$$
\begin{aligned}
& \therefore \mathrm{x}=f \lambda t \\
& \Rightarrow x=\frac{f h}{p} t\left[\lambda=\frac{h}{p}\right] \\
& =>x p=h f t \\
& \therefore x p=h f t
\end{aligned}
$$

This is the principle of certainty. By this formula it is possible to accurately determine the momentum and position of an object.

Method (2) ( principle of certainty according to the theory of relativity): 
$x p=h f t$

The above formula is a combination of Newton's formula.Now let us see the proof of the principle of certainty according to the special relativity theory.

Suppose your size is equal to an electron.You will go to Dhaka bus stop in a car at fixed velocity. The car you are sitting in is shaped like a hydrogen atom. Your friend is also shaped like an electron and he is standing $\mathrm{x}$ meters away from the bus stop.You are going to the bus stop at $v$ velocity. After a while your distance from the bus stop is $x$ meters. That means at that moment you and your friend are at the same distance from the bus stop.The distance and time of the two are being calculated from this very moment. Now the bus stop is $x$ meters away from you and the bus stop is $x$ meters away from your friend.Since then the time for the two of you has started from 00. Your friend is fixed so his position will not change over time. The distance between your friend and the bus stop is $\mathrm{x}$ meters. The distance between you and the bus stop will decrease $x^{\prime}$ over time as you are going at fixed velocity. The distance between you and the bus stop,

$\mathrm{x}^{\prime}=\mathrm{x}-\mathrm{vt}$

Now the speed of your car may be closer to the speed of light. So according to Lorentz's conversion,

$$
\therefore x_{1}^{\prime}=\frac{x_{1}-v t}{\sqrt{1-\frac{v^{2}}{c^{2}}}}
$$

$\Rightarrow x_{1}{ }^{\prime} m_{0}=\frac{m_{0}}{\sqrt{1-\frac{v^{2}}{c^{2}}}}\left(x_{1}-v t\right)$

$\Rightarrow x_{1}^{\prime} m_{0}=m\left(x_{1}-v t\right)$

$$
\left(m=\frac{m_{0}}{\sqrt{1-\frac{v^{2}}{c^{2}}}}\right)
$$

$=>x_{1}{ }^{\prime} m_{0}=m x_{1}-m v t$

$x_{1}{ }^{\prime} m_{0}+m v t=m x_{1}$

Multiply by $\mathrm{C}$ on both sides of Equation 6.

$\therefore x_{1}^{\prime} m_{0} c+m v c t=x_{1} m c$

$=>x_{1}{ }^{\prime} m_{0} c+p x=x_{1} m c[m v=p, c t=x]$

Now the bus is going at fixed velocity so, 


$$
\begin{aligned}
& t=\frac{x_{1}}{v} \\
& \therefore x_{1}{ }^{\prime} m_{0} c+p x=m c \frac{x_{1}}{v} v \\
& \Rightarrow x_{1}{ }^{\prime} m_{0} c+p x=p c t \quad\left(t=\frac{x_{1}}{v}, p=m v\right) \\
& \Rightarrow x_{1}{ }^{\prime} m_{0} c+p x=E t \quad(E=p c) \\
& x_{1}{ }^{\prime} m_{0} c+p x=h f t \quad(E=h f)
\end{aligned}
$$

Now the bus was at fixed velocity so,

$x_{1}=v t$

$$
\therefore x_{1}-v t=0
$$

From equations (6) and (8) we get,

$$
\begin{gathered}
x_{1}{ }^{\prime}=\frac{x_{1}-v t}{\sqrt{1-\frac{v^{2}}{c^{2}}}} \\
x_{1}{ }^{\prime}=0 \quad\left[x_{1}-v t=0\right]
\end{gathered}
$$

We get from Equation (3), "

$$
\begin{aligned}
& x_{1}{ }^{\prime} m c+p x=h f t \\
& =>p x=h f t \quad\left[x_{1}{ }^{\prime}=0\right] \\
& \therefore x p=h f t
\end{aligned}
$$

This is the "certainty Principle".

Method (3) Certainty principle From the principle of uncertainty : 
Suppose we have two numbers $p$ and $q$.

We do not know the exact value of $p$ and $q$. However, we assume that if the value of $p$ and $q$ is close to 10 , it will be close to the correct value. Now we are given an equation. That is the product of $p$ and $q$ is 10 i.e. $p q=10$. From this equation, if we get close to the right value of $p$, we will move away from the right value of $q$, and if we get close to the right value of $q$, we will move away from the right value of $p$. That is, if you know one value, the value of the other will be wrong. The multiplication of $p$ and $q$ can be 10 in many ways.

$$
\begin{aligned}
& p q=10 \\
& 10 \times 1=10 \\
& 5 \times 2=10 \\
& 1 \times 10=10 \\
& 2 \times 5=10
\end{aligned}
$$

Now if we multiply a constant number by the product of $p$ and $q$ then we can determine the exact value of $p$ and $q . p q k=10, k$ is a variable constant, the value of which gives the exact value of $p$ and $q$. In the same way, in the uncertainty principle, it is possible to determine the momentum and position by multiplying $k$ constant by the values of $p$ and $x$. Let us assume We have been able to invent a device in the laboratory that can simultaneously measure the momentum and position of an electron. Since $\Delta p$ and $\Delta x$ are certain then something must be uncertain according to Heisenberg's uncertainty. Then the value of the constant called $k$ will be uncertain. Then the uncertainty equation of momentum and position will be $\triangleleft x \triangleleft p \times k=\hbar$. Again the uncertainty equation of energy and time will be $\triangleleft E \triangleleft t \times k=\hbar$. Now the two equations express the same meaning differently.So we get from two equations,

$$
\begin{aligned}
& \frac{\triangleleft x \triangleleft p \times k}{\triangleleft E \triangleleft t \times k}=\frac{\hbar}{\hbar} \\
& \Rightarrow \triangleleft x \triangleleft p \times k=\triangleleft E \triangleleft t \times k
\end{aligned}
$$

Here $\mathrm{k}$ is the same constant in both cases and since the value of uncertainty is k constant we can write uncertain momentum and position as the principle of certainty.

$$
\begin{aligned}
& x p=E t \\
& \therefore x p=h f t \quad[E=h f]
\end{aligned}
$$

This is the principle of certainty from the principle of uncertainty. If we notice, we will see that the dimensions are the same for both sides in the certainty principle . 
(6) Mathematical proofs of other formulas from certainty mechanics:

(i) Bohr's angular momentum formula:

Bohr's angular momentum ${ }^{L}=\frac{n h}{2 \pi}$;

Bohr provided the formula for angular momentum based only on conjecture. Bohr had no evidence to support this. Drew Broglie later proved the formula for angular momentum from his formula.At that time there was no doubt in Bohr's formula. Now I will try to prove the formula in a different way.

Heisenberg's uncertainty principle, the uncertainty of position and momentum, $\triangleleft x \triangleleft p \geq \frac{\hbar}{2}$

Similarly the principle of uncertainty of angular momentum and angular position:

When the object rotates along the radius $r$ of the circle with the velocity $p$ then we can write $\Delta \mathrm{x}=\Delta \theta \mathrm{r}$ and $p=\frac{\triangleleft L}{r}$ if the angular momentum is $\mathrm{L}=\mathrm{mv} . \mathrm{r}=\mathrm{p} . \mathrm{r}$ and the angular position is $\theta=\frac{x}{r}$. Now the principle of uncertainty of angular position and angular momentum,

$$
\begin{aligned}
& \triangleleft x \triangleleft p=\frac{h}{2 \pi} \\
& =>\triangleleft \theta r \frac{\triangleleft L}{r}=\frac{h}{2 \pi} \\
& \therefore \triangleleft \theta \triangleleft L=\frac{h}{2 \pi}
\end{aligned}
$$

In the same process there will be a principle of certainty between the angular momentum and the angular position according to the principle of certainty. If

$x=r \theta$ and $p=\frac{L}{r}$ then the certainty principle between angular momentum and angular position will be,

$$
\begin{aligned}
& x p=h f t \\
& \Rightarrow \theta r \frac{L}{r}=h f t
\end{aligned}
$$

$$
\therefore \theta L=h f t
$$

This is the principle of certainty between angular momentum and angular position.

This time I will prove the formula of Bohr's angular momentum from the certainty principle of angular momentum and angular position.

$\theta L=h f t$ is the principle of certainty of the angular position and angular momentum of a particle during rotation in a circle. Now we get from here, 
$\omega t L=h f t$

$\Rightarrow \omega L=h f$

From the above equation it can be seen that the product of angular momentum and angular velocity is related to the Planck formula. That is, the product of angular momentum and angular velocity is equal to the energy $E$. Let's arranged this equation a little bit,

$\omega L=h f$

$=>\omega L=h \frac{c}{\lambda}$

Now hold the velocity of the particle equal to the velocity of light. If $\omega=\frac{c}{r}$,

$\frac{c}{r} L=\frac{h c}{\lambda}$

$=>\frac{\lambda}{r} L=h$

Now the particle rotates around the circle. So if the wavelength of the wave involved is $\lambda=\frac{2 \pi r}{n}$,

$\therefore \frac{2 \pi r}{n r} L=h \quad\left[\lambda=\frac{2 \pi r}{n}\right]$

$\therefore L=\frac{n h}{2 \pi}$

This is Bohr's formula for angular momentum. The law of angular momentum can be determined accurately from the "principle of certainty".

Thus the laws of physics are intertwined.

There is no uncertainty inside Bohr's angular momentum. The principle of certainty is similar to the angular momentum. The more accurately the radius $(r)$ of orbit can be determined from Bohr's law, the more accurately it is possible to determine the momentum of an electron.

(ii) Evidence of the Max Planck formula : The formula $E=$ hv of quantum theory can be proved from the principle of certainty. Now we will prove this formula from the principle of certainty. In subject to $v$ let us differentiate $E=1 / 2 \mathrm{mv}^{2}$,

$$
\Delta \mathrm{E}=\mathrm{mv} \Delta \mathrm{v}
$$

Now let's integrate this equation ,

$$
E=1 / 2 m v^{2}
$$

We get from the equation of integration,

$\Delta \mathrm{E}=\mathrm{p} \Delta \mathrm{v}$ or $\boldsymbol{p}=\frac{\triangleleft E}{\triangleleft v}$. From the equation we put the value of $\mathrm{p}$ at the principle of certainty $\mathrm{xp}$ $=\mathrm{hft}$, 


$$
\begin{aligned}
& x \frac{\triangleleft E}{\triangleleft v}=h f t \\
& \Rightarrow x \triangleleft E=h f t \triangleleft v
\end{aligned}
$$

Now let's integrate this equation.

$$
\begin{aligned}
& E x=h f t v \\
& \Rightarrow E x=h f x \quad[x=v t] \\
& \therefore E=h f
\end{aligned}
$$

(iii) Bohr's formula for angular momentum:

The relationship between Einstein's photoelectric effect and Bohr's energy radiation can be established by the mechanics of certainty. If an electron is pushed by $F$ force at energy level 1 , it will go to 2 . The distance between the energy levels 1 and 2 is ds. We get from the second formula,

$F=\frac{h}{c} \frac{d f}{d t}$

When the velocity of the object is $v$,

$$
F=\frac{h}{v} \frac{d f}{d t}
$$

Now let us bring out the total energy ,

$$
\begin{aligned}
& W=\int_{u}^{v} F d s \\
& =>E_{k}=\frac{h}{v} \int_{u}^{v} \frac{d f}{d t} d s \quad\left(F=\frac{h}{v} \frac{d f}{d t}\right) \\
& =E_{k}=\frac{h}{v} \int_{u}^{v} \frac{d s}{d t} d f \\
& =>E_{k}=\frac{h}{v} \int_{u}^{v} v d f \\
& =>E_{k}=h \int_{f_{1}}^{f_{2}} d f \\
& =>E_{k}=h[f]_{f_{2}}^{f_{1}} \quad\left(v=\sqrt{\frac{h f}{m}}\right) \\
& =E_{k}=h f_{2}-h f_{1}
\end{aligned}
$$


Now when an electron moves from one energy level to another, the electron will give up or receive $E=h v$ amount of energy from the total energy. Then the total energy becomes kinetic energy and goes to another energy level. So we can write,

$\Rightarrow E=h f_{2}-h f_{1}$

$$
\therefore h v=E_{2}-E_{1}
$$

\section{(iv) Evidence of uncertainty principle:}

\section{Method: 1}

Many people may feel uncomfortable seeing the principle of certainty. This is very natural because for the last 100 years everyone has come to believe that the principle of uncertainty is actually part of nature. Einstein said, "Something is happening wrong and that's why this uncertainty has come." Now I will tell you why uncertainty comes. Suppose A snake is running in the river. At that time waves are created in the river. Now if I find the snake inside the wave then the position of the snake will be uncertain. But if I find a snake in a river without waves, there will be no uncertainty. A particle is moving. Waves are created or waves are involved because of the motion of the particles. The waves are like beautiful sine waves. Like a snake, we will look for the particle inside the wave, that is, inside the sine function.

The wave is $\sin (\omega t)$

Now the principle of certainty is,

$x p=h f t$

$\Rightarrow x p=\frac{h}{2 \pi} 2 \pi f t$

$=>x p=\hbar \omega t$

$\omega t=\frac{x p}{\hbar}$

We get from equations (9) and (10);

$\sin \left(\frac{x p}{\hbar}\right)$

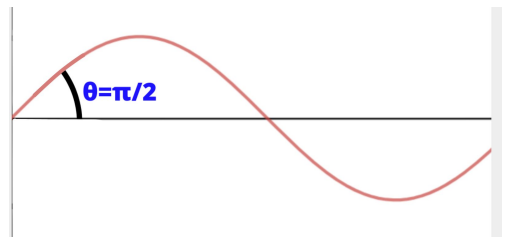

Figure 1: An electron at the main point and the wave created due to the momentum of the electron are depicted. 
The value of the wave is, $-1 \leq \sin (\omega t) \leq 1$

But $x p=$ will not be a negative value.

That is,

$\theta \geq 0$

$=>\frac{x p}{\hbar} \geq 0$

So hold the distance $\theta=\omega t \geq \frac{\pi}{2}$ between the electron and the wave at the original point.

$$
\begin{aligned}
& \therefore \frac{x p}{\hbar} \geq \frac{\pi}{2} \\
& =>x p \geq \frac{\pi}{2} \times \frac{h}{2 \pi} \\
& =>x p \geq \frac{h}{4} \\
& \therefore \triangleleft x \triangleleft p \geq \frac{h}{4}
\end{aligned}
$$

Which indicates uncertainty. It turned out to be a call to God in the face of demons. I said a while ago that the principle of certainty is correct. Again I am saying, if the certainty is correct, then the principle of uncertainty is also correct.

In fact, the reason for the uncertainty is that I found the electron in $\sin (\theta)$ at $\theta \geq \pi / 2$. That is, when I look for electrons inside the wave, uncertainty will come. If we continue to reduce the value of $\theta$, then the value of uncertainty will continue to decrease. In this way uncertainty can be removed. If the value of $\theta$ reduces to 0 , that is, if the distance between the electron and the wave created by the electron is $\theta=0$ then there is nothing to say about uncertainty. What is uncertainty? Is it very important to stay?

Can't it be omitted in any way? What is the role of the certainty from which the uncertainty came? Will we not get rid of uncertainty in any way?

Dolphins are running in the sea. For convenience, there is no wave in the sea.

Now when dolphins run, they create waves. The size of each wave is equal to 10 to 20 floors (imagine, there is nothing wrong with imagining!). Now the dolphin is running through waves equal to 10 to 20 floors. I don't understand where the dolphins are. Now you have to look at the sea waves to identify the dolphins. Then we can say that the bigger the wave, the more likely it is that there will be dolphins. when we can't identify the dolphin, instead we go to identify the dolphin by the waves, then the dolphins are everywhere in the waves of the ocean. Then the uncertainty will come.Similarly, when we cannot identify the electron, when we go to determine the position of the electron through the created (involved) wave due to the movement of the electron, then uncertainty will come. Whether it's finding the dolphin in the waves of the ocean or finding the electron in the waves created with it. The dolphin or the electron is in a certain position but the waves created with it can be in any position. Just then uncertainty shifts from certainty. And if we do not want to bring uncertainty, then the distance between the electron and the created (involved) wave with the electron must be calculated as $\theta=0$. Then there will be no uncertainty. 
I have done a little trick in implementing the principle of uncertainty from the principle of certainty.

$$
\sin \left(\frac{x p}{\hbar}\right)
$$

Here we have calculated holding $\frac{x p}{\hbar} \geq \frac{\pi}{2}$.

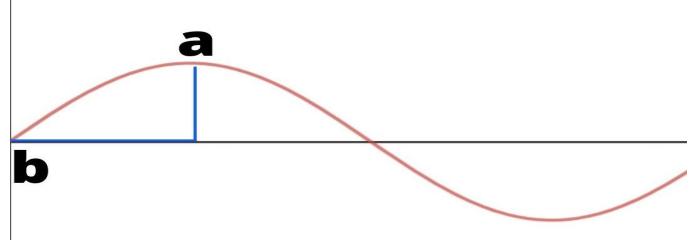

We have calculated from the highest peak in the figure i.e. $\theta=\pi / 2^{\circ}$ but we should have calculated from $\theta=0^{\circ}$ first.

Let's calculate the beginning with ${ }^{\circ}=0$.

I have said before that if you reduce $\theta$, the value of uncertainty will continue to decrease. So what will happen now?

Suppose the distance between an electron and a wave created with an electron is $\theta=0^{\circ}$.

$$
\frac{\triangleleft x \triangleleft p}{\hbar} \geq 0
$$

$\therefore \triangleleft x \triangleleft p \geq 0$

But now there is no uncertainty.

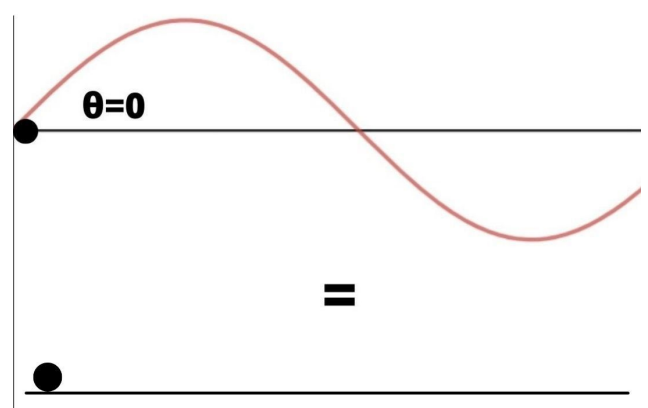

\section{Method 2}

Let's find out the general equation of the relationship between the principle of certainty and the principle of uncertainty. 


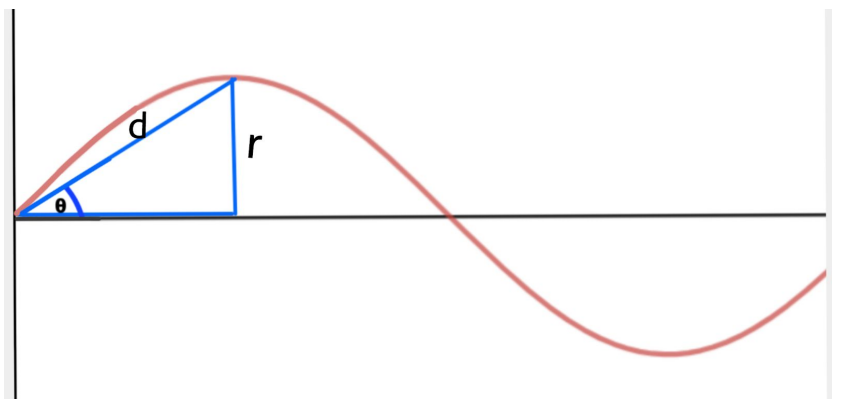

Notice the figure above which is the graph for $\sin (\theta)$. Here $\sin (\theta)=r / d$.

$\sin (\theta)=\frac{r}{d}$

$=>\sin (\omega t)=\frac{r}{d}$

Now we know, $\omega t=\frac{x p}{\hbar}$.

$\therefore \sin \left(\frac{x p}{\hbar}\right)=\frac{r}{d}$

$=>\frac{x p}{\hbar}=\sin ^{-1}\left(\frac{r}{d}\right)$

Now if we know the momentum $p$ then the uncertainty of position will be ,

$\triangleleft x=\frac{\hbar}{p} \sin ^{-1}\left(\frac{r}{d}\right)$

The reason for the uncertainty is that if the distance between the electrons and the waves associated with the electrons (created waves) is calculated more, the uncertainty comes. Whether the uncertainty will be more or less will depend on the following equation.

$\triangleleft x \propto \sin ^{-1}\left(\frac{r}{d}\right)$

The mystery of whether uncertainty will increase or decrease is hidden in this equation.

I already told why the uncertainty came.

Let's take a look at the relationship between the principle of uncertainty and the principle of certainty according to Broglie's particle-wave duality.

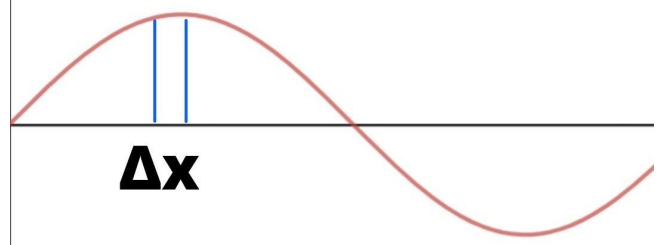


If we are sure of $\Delta x$ then we do not know what will be $\lambda$. Then according to the $p=\frac{h}{\lambda}$ formula $\Delta x$ is sure but the momentum $\mathrm{p}$ is uncertain.

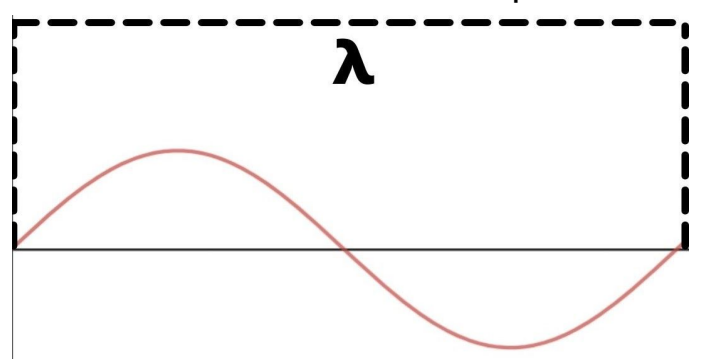

Now $\lambda$ confirmation means that according to the formula $p=\frac{h}{\lambda}$ the momentum $\mathrm{p}$ is certain but $\Delta \mathrm{x}$ is then uncertain. Then what is the matter!

We have seen from the principle of certainty that if it is $\theta \geq \pi / 2$ then uncertainty comes. Which means I've been trying to find the electrons inside the waves. $\lambda$ Confirmation in the Broglie's formula and $\theta \geq \pi / 2$ in the principle of certainty is the same thing. Then this is the background behind the coming of uncertainty.

7.Decision: If the distance between the electron and the wave created by the electron is $\theta=$ 0 then there will be no more uncertainty. Uncertainty increases when the distance difference between the electron and the generated wave increases. For certainty we need to take the distance $\theta=0^{\circ}$ between the electrons and the waves involved due to the movement of electrons. When we look for particles inside the wave then we will not know where the particle's position is. Then you just have to figure out the possibility of where the particle will be from Schrodinger's equation. If we know the momentum of the particle and the frequency of the waves created with it, we will be able to determine the position of the particle after $t$ times from the principle of certainty. Does the principle of certainty prove the principle of uncertainty wrong ? The answer is no. If the distance between the particle and the wave is $\theta \geq \pi / 2$ then uncertainty will come.In fact, the principle of certainty defends the principle of uncertainty .In fact, the principle of uncertainty got a new life. Why the uncertainty comes is now sure . Quantum entanglement has a faster effect than light to determine the momentum and position of two particles. Now let's try to solve this problem from quantum certainty mechanics. IFirstly, electrons begin to move with a certain momentum So the waves get involved with the particles. When no external force is applied, the frequency of the waves associated with the particle remains constant. The frequency of the waves involved is constant after the two particles collide with each other. So even though many light-years away, the behavior of the two particles is similarly inverse due to the fact that the frequency is constant. After applying force to each other, the particle's momentum and position will behave according to the conservation law of energy at a distance of many light years. In this case, Super Determinism will be proved wrong.Through the conservation law of energy in quantum entanglement, it is possible to determine the results of two particles many light years away. Electrons are particles and when electrons move with the momentum $p$ then waves are created with them ( or waves are involved). Quantum mechanics can be protected from terrible stems by calculating the waves and particles involved in the electrons separately. At the time of observing the electrons in the laboratory, if we calculate the 
external force by the laboratory instrument, then the definite momentum and position can be determined.

8. Conclusion: Quantum certainty mechanics is a theory that can mathematically prove many principles of quantum mechanics, including the Uncertainty principle, Max Planck's quantum theory. Einstein said until his death that there was a reason behind the uncertainty. Uncertainty comes from what can be perfectly explained by the principle of certainty. Uncertainty is an important formula of quantum mechanics which is $100 \%$ correct and if uncertainty principle is $100 \%$ correct then certainty principle is also correct. The principle of uncertainty has not been proved wrong by the principle of certainty but the principle of certainty has revived the principle of uncertainty. The principle of certainty and the principle of uncertainty complement each other. Certainty mechanics has been invented to protect quantum mechanics from terrible shoots. There is a clear idea from the mechanics of certainty that calculating $\theta \geq \pi / 2$ leads to uncertainty. The most complex problems in quantum mechanics can be solved from quantum certainty mechanics. Quantum certainty mechanics gives an idea of how much energy a photon changes due to electron displacement. The essence of certainty mechanics is, "Knowing the frequency of the waves involved in a particle, the momentum and position can be determined simultaneously over time."

\section{Reference:}

[1] Ozawa, M: Heisenberg's Original Derivation of the Uncertainty Principle and its Universally Valid Reformulations (2015).arXiv:1507.02010

[2] https://en.wikipedia.org/wiki/Uncertainty_principle.

[3] Heisenberg, W. The physical content of quantum kinematics and mechanics. In Wheeler, J. A. \& Zurek, W. H. (eds.) Quantum Theory and Measurement, 62-84 (Princeton UP, Princeton, NJ.

[3]Ozawa, M. Noise and disturbance in quantum measurements and operations. Proc. SPIE 6244, 62440Q (2006).

[4] Lund, A. P. \& Wiseman, H. M. Measuring measurementdisturbance relationships with weak values. New J. Phys. 12, 093011 (2010).

[5] Ozawa, M. Uncertainty relations for noise and disturbance in generalized quantum measurements. Ann. Phys. (N.Y.) 311, 350416 (2004).

[6] J. D. Walecka, Introduction to Modern Physics: Theoretical Foundations, World Scientific, 2008, pp. 477.

[7] Pavle I. Premović , P.I: The Energy-Position and the Momentum-Time Uncertainty Expressions (2018)

[8] M. Planck, Verh. Dtsch. Phys. Ges. 1900, 2, 237; M. Planck, Ann.

Phys. 1901, 4, $553-564$.

[9] A. Einstein, Ann. Phys. 1905, 17, 132 - 184. 
[10]N. Bohr, Nature 1923, 112, $29-44$.

[11] N. Bohr, Philos. Mag. Ser. 6 1913, 26, 1 - 25; N. Bohr, Philos.

Mag. Ser. 6 1913, 26, 476 - 502; N. Bohr, Philos. Mag. Ser. 6 1913,

26, 857 - 875; b) N. Bohr, Nature 1913, 92, 231 - 232.

[10] https://en.wikipedia.org/wiki/Quantum_entanglement?wprov=sfla1

[11] A. Fine, The Einstein-Podolsky-Rosen argument in quantum theory, The Stanford Encyclopedia of Philosophy (Winter 2014 Edition), Edward N. Zalta (ed.), URL http://plato.stanford.edu/archives/win2014/entries/qt-epr/ .

[12] A. Einstein, B. Podolsky, N. Rosen, Can quantum-mechanical description of physical reality be considered complete?, Phys. Rev. 47 (1935) 777-780.

[13] Michaud, A :On de Broglie's Double-Particle Photon Hypothesis (2016) .

[14] https://en.wikipedia.org/wiki/Matter wave?wprov=sfla1 